\title{
Formation of massive stars at low metallicity: predictions, observations and expectations
}

\author{
Henny J. G. L. M. Lamers \\ Astronomical Institute, Utrecht University, and SRON Laboratory for Space Research, \\ Princetonplein 5, NL-3584CC, Utrecht, The Netherlands \\ email:lamers@astro.uu.nl
}

\begin{abstract}
Star formation at very low metallicity is expected to produce only massive stars. This is a result of the low cooling rate. Hydrodynamical simulations of star formation from zero metallicity gas suggest that the first stars had masses in excess of about $10^{2} M_{\odot}$. These stars can not be observed because of their large redshift and their very short lifetime. However, a similar (but not the same) effect might be observable in regions of star formation close to very luminous radiation sources, where intense radiation may destroy dust and CO molecules in starforming clouds. Such clouds will be warmer than in normal metal rich star forming regions because of the reduced cooling, which is then predominantly due to $\mathrm{H}_{2}$ and atomic $\mathrm{C}$ and $\mathrm{O}$. Under those conditions star formation may result in the formation of high mass stars without the normal large numbers of accompanying low mass stars. Observations show that this process may occur close to the centre of the interacting galaxy M51, where the intense radiation of the nuclear starburst and the small dust content destroyed molecular $\mathrm{CO}$ in star forming regions. HST observations of this region show the presence of about 30 massive stars, of $25<M<150$ $M_{\odot}$, without the accompanying clusters of low mass stars.
\end{abstract}

Keywords. Stars:evolution, Stars: formation, Galaxies: stellar content, Galaxies: individual (M51)

\section{Introduction}

Understanding the formation of zero metallicity stars is important because of its enormous consequences, in particular in terms of the reionization of the Universe and the early enrichment of the galactic and intergalactic medium. In the last few years we have witnessed a dramatic progress in this field, due to hydrodynamical simulations which are now capable of following the process on scales from megaparsecs down to stellar scales (see e.g. Abel et al. 2000, 2002; Bromm et al. 1999, 2002, Clarke \& Bromm 2003; Yoshida et al. 2003). These studies have shown that the characteristic mass of stars to be formed depends strongly on the metallicity. The reason for this is simple:

1. Clouds can self-gravitate if their mass exceeds the Jeans mass

2. The Jeans mass depends on the density and temperature as $M_{J} \sim \rho^{-1 / 2} T^{3 / 2}$

3. The temperature of a cloud during its contraction depends on the cooling efficiency which depends on metallicity

4. Hence the characteristic stellar mass depends on metallicity.

The Jeans mass is

$$
M_{J}=\left\{\pi k / G m_{H} \mu\right\}^{3 / 2} T^{3 / 2} \rho^{-1 / 2} \simeq 25 T^{3 / 2} n^{-1 / 2} \quad M_{\odot}
$$

where $n$ is the particle density of $\mathrm{H}_{2}$ in $\mathrm{cm}^{-3}$. For a cloud of $n=10^{6} \mathrm{~cm}^{-3}$ the Jeans mass is $0.8 M_{\odot}$ if $T=10 \mathrm{~K}$ (the characteristic temperature for galactic clouds) and 70 $M_{\odot}$ if $T=200 K$ (the characteristic value for a metal-free cloud). 
Unfortunately, the first stars were formed at high redshift, $z>15$, and had a very short lifetime of only a few Myr. So their formation process cannot be witnessed with present day telescopes. However, it turns out that the formation of massive stars may occur in an analogous (but not the same) way under special conditions when radiation form a nearby intense source destroys the CO-molecules in star forming regions and hence reduces their cooling rate drastically.

We stumbled upon such a case when studying the nucleus of the interacting galaxy M51 with HST. The old and "yellowish" region around the nucleus, which we will call the "Bulge" (although M51 is seen almost face-on), contains a few dozens of mainly blue point sources. A study of these sources suggests that they are point sources and their energy distribution suggests that they are isolated or small groups of massive stars rather than low mass clusters.

I will briefly discuss the predicted star formation from metal-free clouds, the properties of the massive stars in the bulge of M51, and the possible explanation why star formation near the nucleus of M51 may somehow ressemble that in the early Universe.

\section{Predicted formation of low metallicity stars}

The formation of metal-free stars has been simulated using SPH methods by several groups (Haiman et al. 1996, Haiman \& Loeb 1997; Abel et al. 2000, 2002; Clarke \& Bromm 2003) and has been recently reviewed in an excellent paper by Bromm \& Larson (2004). The main effects can be seen in Figure 1 (from Bromm, Coppi \& Larson 2002), which shows the gas properties of SPH particles of a metal-free gas that started to collapse in a CDM Universe at redshift $z=31.2$.

The temperature distribution shows two distinct branches. At densities below about $1 \mathrm{~cm}^{-3}$ the $\mathrm{H}_{2}$ fraction is very small, $<10^{-5}$, and the cooling of the contracting clouds is very inefficient. As a consequence the temperature rises almost adiabatically as the gas contracts, i.e. $T \sim n^{2 / 3}$, until it reaches the virial value of $\sim 5000 \mathrm{~K}$ at that redshift. At densities above about $1 \mathrm{~cm}^{-3}$ the molecular fraction increases and cooling by $\mathrm{H}_{2}$ molecules becomes important. This results in an almost isobaric decrease of temperature when the density rises, $T \sim n^{-1}$. $\mathrm{H}_{2}$ can cool the gas down to about $T \simeq 500 \mathrm{~K}$. At that point the gas reaches a quasi-hydrostatic state with a density of about $n \simeq 10^{4} \mathrm{~cm}^{-3}$. (The minimum temperature reached by $\mathrm{H}_{2}$ cooling is due to the fact that the lowest excited rotational level of the $\mathrm{H}_{2}$ molecule has an energy of $E / k \simeq 500 \mathrm{~K}$. So when $T$ drops below this value, collisions can no longer excite $\mathrm{H}_{2}$ to this level and the cooling stops. In practice, collisions with particles in the tail of the Maxwell velocity distribution can cool $\mathrm{H}_{2}$ gas down to about $200 \mathrm{~K}$.)

For every value of the density and temperature the corresponding Jeans mass can be calculated (Eq.1). The result is shown in the lower right panel of Fig. 1. The Jeans mass of the quasi-hydrostatic clumps of gas of $T \simeq 500 \mathrm{~K}$ and $n \simeq 10^{4} \mathrm{~cm}^{-3}$ is about $10^{3} M_{\odot}$. The onset of gravitational instability can compress the gas further, with a modest rise in $T$ and a decrease in $M_{J}$ down to about $200 M_{\odot}$, which is the limiting resolution of these simulations.

The numerical simulations of subsequent star formation have shown that the resulting characteristic mass of stars to be formed out of metal-free gas is of the order or slightly smaller than this value of the Jeans mass, so the first stars are expected to have masses of order $10^{2} M_{\odot}$ (e.g. Clarke \& Bromm 2003). The minimum stellar mass is set by the mass of the fragments for which the cooling rate is higher than the rate at which gravitational energy is released during the collapse. 

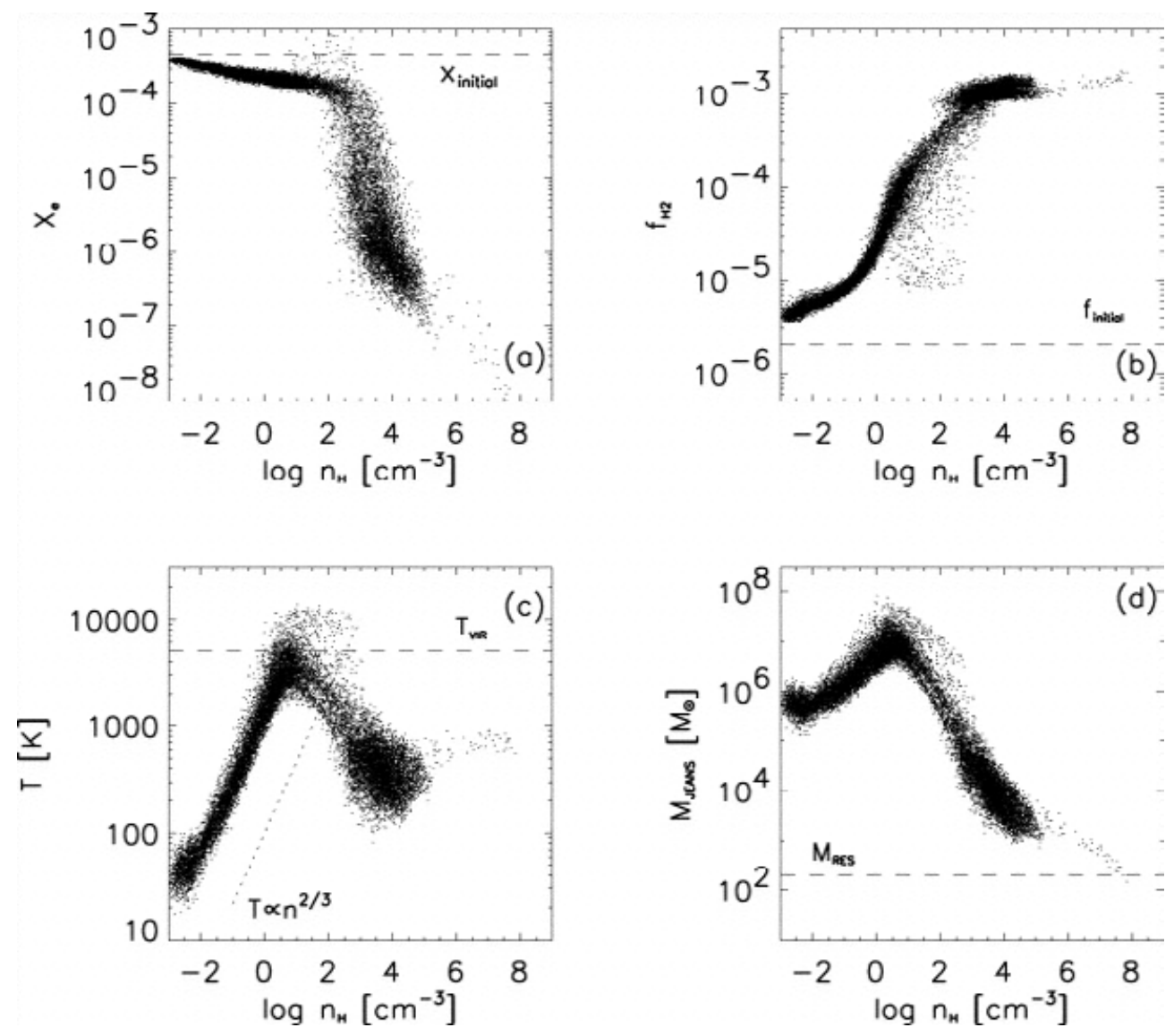

Figure 1. The properties of metal-free gas at redshift $z \simeq 30$ as a function of their density. Upper left: the fraction of free electrons. Upper right: the abundance of molecular hydrogen. Lower left: the temperature. Lower right: the corresponding Jeans mass. (from Bromm, Coppi \& Larson 1999)

Numerical simulations also show that there is a critical metallicity for star formation with a high characteristic mass. If the metallicity is lower than $Z / Z_{\odot} \simeq 10^{-3.5}$ the characteristic mass is high, of order $10^{2} M_{\odot}$. If the metallicity is lower then the characteristic mass is about $1 M_{\odot}$ (Clarke \& Bromm 2003).

Figure 2 shows the characteristic mass of stars as a function of redshift for two cases: with and without $\mathrm{CO}$. In one case the cooling is by $\mathrm{H}_{2}$ molecules only, in the other case the cooling is dominated by $\mathrm{CO}$ molecules. Notice that in the local Universe, near $Z \leqslant 1$, clouds without CO-cooling produce stars with a characteristic mass of order 50 to 500 $M_{\odot}$ (depending on the initial overdensity) whereas CO-cooling results in a characteristic mass between 0.2 and $1 M_{\odot}$.

\section{The formation of massive stars near the nucleus of M51}

\subsection{The observed point sources: low mass clusters or massive stars?}

The process of star formation at very low metallicity in the early Universe, described above, cannot be observed with present day instrumentation: the distance is too large. Moreover the massive stars formed in this process live only a very short time of order 


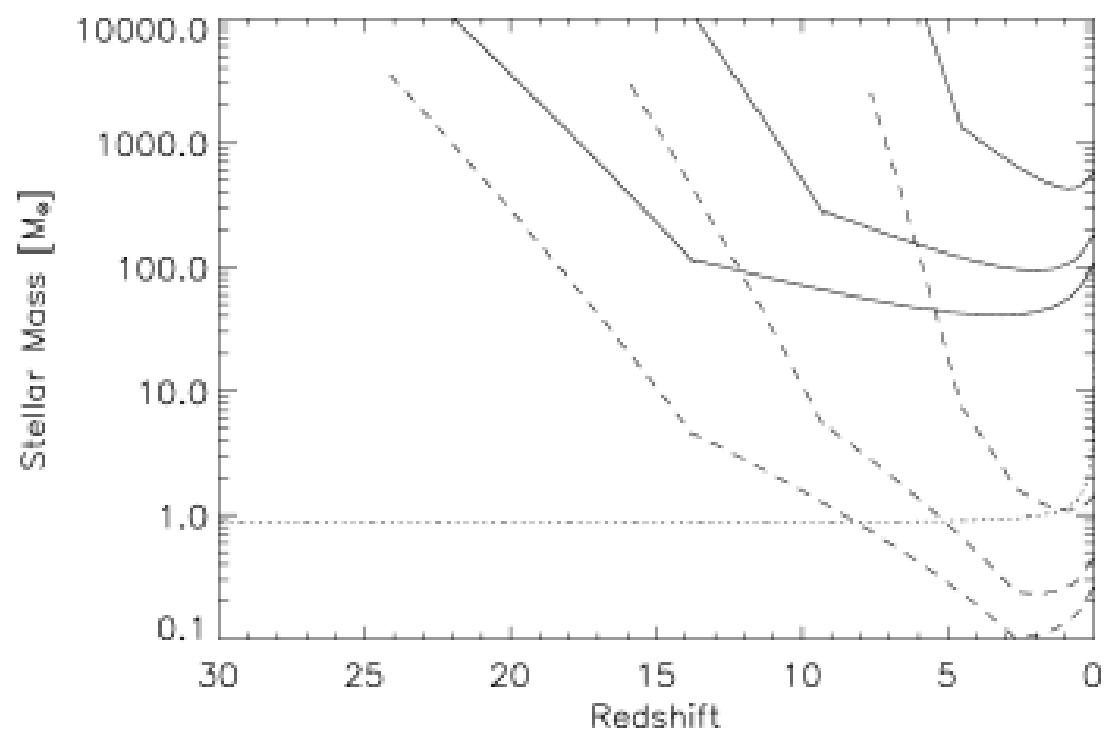

Figure 2. The characteristic mass of stars as a function of redshift. The full lines show the mass for models with $\mathrm{H}_{2}$-cooling for initial density fluctuations of 1,2 and $3 \sigma$ (from right to left). The dashed lines show the predicted mass that results from clouds with CO-cooling, for the same density fluctuations. The characteristic stellar mass in the local Universe, at redshift $z \leqslant 1$, is 50-500 $M_{\odot}$ for $\mathrm{H}_{2}$-cooling and 0.3-1 $M_{\odot}$ for CO-cooling. The dotted line shows the minimum mass of stars formed at redshift $z$ that is observable at present. (from Clarke \& Bromm 2003).

a few Myr. However, there may be locations where the conditions are so special that the process of star formation may be similar, although not the same, as in the very low metallicity era. The ongoing formation of massive stars near the nucleus of the interacting Whirlpool galaxy (M51) may occur at such a location.

M51 is a grand-design spiral galaxy that interacted with its companion about 400 Myr ago (Toomre \& Toomre 1972; Hernquist, 1990; Barnes 1998) and possibly also more recently 50-100 Myr ago (Salo \& Laurikainen 2000). The nucleus of M51 harbors a huge starburst with an age of about $400 \mathrm{Myr}$, a luminosity of $1.310^{8} \mathrm{~L}_{\odot}$ and a mass of $2.210^{7} M_{\odot}$ (Panagia et al. 2001; Scuderi et al. 2005). The bulge of M51 contains very little dust, with a maximum extinction of about 0.25 in $E(B-V)$ (Lamers et al. 2002). The HII regions of M51 have a metallicity of about twice solar.

The HST - PC image of the central region of M51 in the $F 439 W$-band shows the presence of bright pixel-size spots, with $21.4<V<24.4$ (Fig. 3). These were observed in six filters, from $U V$ to $I$ and studied by Lamers et al. (2002). The sizes of the sources were estimated, using radial profile fitting of the $F 555 \mathrm{~W}$ images. The vast majority, 21 out of 30 , are definitely point sources. Six sources have a too high background to allow a reliable size determination. Two sources are possibly extended, but they have a low peak countrate and are rather faint. So the majority of the sources are pointlike with a FWHM less than about $0.3 H S T-P C$ pixels, which corresponds to $0.6 \mathrm{pc}$ at the distance of $8.4 \mathrm{Mpc}$ of M51. If the sources are normal star clusters, their masses are in the range of $310^{2}$ to $310^{3} M_{\odot}$ for the younger ones of $t \leqslant 30 \mathrm{Myr}$ and $10^{4}$ to $10^{5} M_{\odot}$ for the older ones of $t \geqslant 500 \mathrm{Myr}$. If the sources are stars, their luminosities are in the range of about $4.5<\log \left(L / L_{\odot}\right)<7.0$ and their ages are less than $10 \mathrm{Myr}$.

The location of the sources is shown in Fig. 3. Their distance to the nucleus of M51 is between 90 and $270 \mathrm{pc}$. They are located in strings that follow the general pattern of 


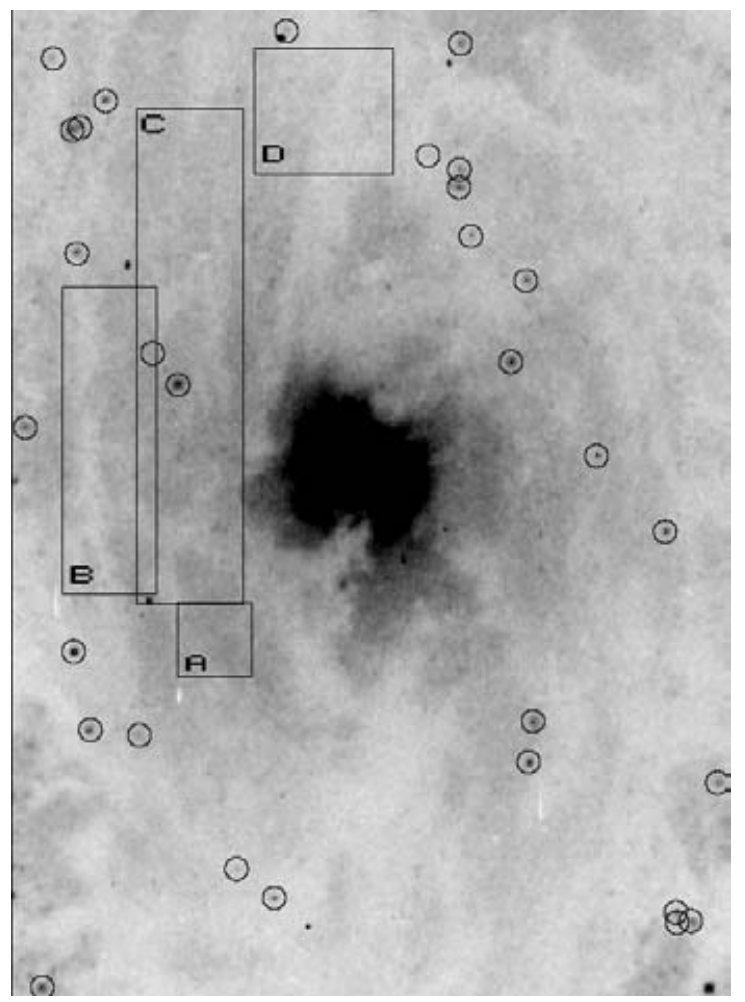

Figure 3. The location of the bulge point sources in inner region of M51. Image size: $450 \times 650$ pc. The sources are located in strings that follow the general pattern of the inner extension of the spiral arms. This pattern can also be traced by the very faint dust lanes. It shows that the sources were not ejected from the nucleus but formed in situ. The rectangular regions, A to D, were used for the study of the dust extinction properties. (from Lamers et al. 2002)

the faint dust lanes which trace the inner extensions of the spiral arms of M51. ¿From here-on these sources will be called "bulge point sources".

The energy distribution of the bulge point sources in six HST wavelength bands, from $U V$ to $I$ was compared to that of the Starburst99 cluster models (Leitherer et al. 1999) and to stellar models (Kurucz 1993) using the 3DEF-method of Bik et al. (2003), with three free parameters: $E(B-V)$, age and mass in case of cluster models and $E(B-V)$, $T_{\text {eff }}$ and $R$ in case of stellar models. The energy distributions fit the stellar models significantly better than the cluster models. A detailed study of the energy distribution of the most luminous sources, with the smallest photometric uncertainties, clearly indicates low upperlimits for the number of low mass stars that could be contributing to these sources. So the sources are not "normal" clusters with a normal stellar IMF.

We conclude that the large majority of the bulge point sources of M51 are most likely massive stars or small groups of massive stars, with a complete lack or a severe under representation of low mass stars. (Another way to express this result is: if the sources are clusters, they have a strongly top-heavy IMF). This is confirmed by their location in the HR-diagram, shown in Fig. 4.

In any case, for simplicity we will refer to the sources as "stars", although they may in fact be small groups of massive stars. The location of the stars in the HR-diagram shows a few characteristic properties: 


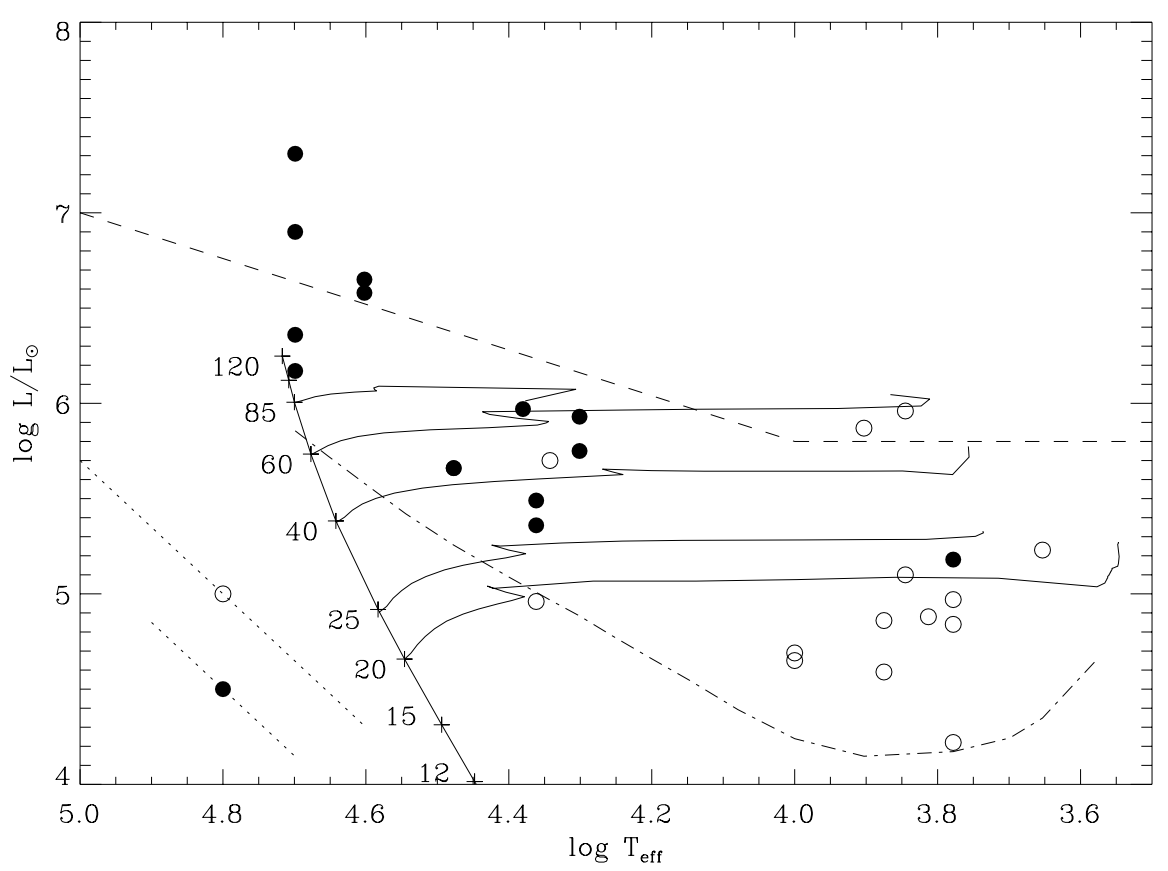

Figure 4. The location of the bulge point sources in the HR-diagram with $T_{\text {eff }}$ and $L$ derived from $H S T$ UV,U,B,V,R,I photometry. Open circles refer to sources not detected in the UV and U band images. Typical error bars are shown in the lower left hand corner. Predicted evolutionary tracks are indicated for different masses. The dashed line is the Humphreys-Davidson luminosity upperlimit. The dash-dotted line shows the detection limit. The location of the sources in this diagram confirms our suggestion that the sources are massive stars or small groups of massive stars, rather than low clusters. (from Lamers et al. 2002)

- Within the uncertainty of the derived values of $L$ and $T_{\text {eff }}$ the luminosity is below the Humphreys-Davidson limit, as expected for stars but not for clusters. (Four of the hottest stars may be brighter than the HD-limit but the uncertainties are large.)

- If the sources are single stars, they are in the mass range of about $20<M<200 M_{\odot}$. There may be lower mass stars, but these would be below the detection limit for single stars.

- There appears to be a lack of sources in the range of $4.0<\log \left(T_{\text {eff }}\right)<4.3$. This is expected for stars because they cross this temperature region on a very short KelvinHelmholtz timescale. (If the sources were clusters instead of stars, their colours would indicate a gap in the age distribution between about 30 and 500 Myr.).

These properties confirm our suggestion that the sources are in fact massive stars or small groups of massive stars. So we conclude that star formation is going on in the bulge of M51 and that massive stars or small groups of massive stars are formed. At this location the massive stars are formed without the large number of low mass stars that usually accompanies massive stars.

\section{Why does the region around the nucleus of M51 form massive stars in isolation?}

What could be the reason for the suppressed formation of low mass stars within a few hundred pc from the nucleus of M51? 
The core of M51 contains a starburst with an age of $410 \pm 140 \mathrm{Myr}$, in agreement with the estimated time of close encounter with companion NGC 5195. The total mass in the core is $\sim 2.210^{7} M_{\odot}$ within the central $17 \mathrm{pc}$ and its luminosity is $1.310^{8} L_{\odot}$ (Panagia et al. 2001; Scuderi et al. 2005). Observations as well as theory shows that this radiation affects the conditions in the ISM surrounding the nucleus.

(a) CO-images of the central region of M51 show a hole in the inner few hundred pc, apart from one large cloud (Aalto et al. 1999, fig. 7). The massive stars are located in this hole.

(b) The dust extinction in the bulge is very small. Even the most reddened pixels in the bulge-regions have an extinction of only $E(B-V) \simeq 0.25$ (see Fig. 3).

(c) Norman \& Spaans (1997) and Mihos, Spaans \& McCaugh (1999) have studied the conditions for the formation of massive stars. They have suggested that isolated massive stars can be formed in clouds in which $\mathrm{H}_{2}$, [OI] $63 \mu \mathrm{m}$ and [CII] $158 \mu \mathrm{m}$ are the dominant coolants. This occurs for instance in the vicinity of a hot ionizing source in a region with an optical depth $A_{V} \leqslant 1$ so that $\mathrm{CO}$ is dissociated but $\mathrm{H}_{2}$ is protected by self-shielding. It is very well possible that these conditions are met in the bulge of M51. (The UV radiation field strength at a distance of about $200 \mathrm{pc}$ from the nucleus of M51 is about $10^{2}-10^{3}$ in units of the mean radiation field in the disk of Galaxy.)

(d) Models calculated by Marco Spaans (Private Communication, see also Lamers et al. 2002) of the thermal and chemical structure of interstellar clouds that have 1 or 2 magnitudes of visual extinction show that CO is largely destroyed and that the [OI] fine structure line at $63 \mu \mathrm{m}$ is the dominant coolant.

(e) The destruction of CO in clouds of small extinction and the increased photo-electric heating will result in relatively high cloud temperatures of 300 to $2000 \mathrm{~K}$. This will lead to high a Jeans mass for gravitational contraction. Moreover, the McKee criterium of $A_{V} \sim 6$ (McKee, 1989) to sustain star formation is probably not satisfied for the bulk of the gas. Since the low extinction causes any stellar source to induce disfavorable conditions for further star formation in its vicinity, isolated patches of massive stars are the natural state. (See also Omukai \& Palla, 2001, 2003 and Tan \& McKee 2004).

\section{Summary, consequences and outlook}

In the region around the nucleus of the interacting Galaxy M51 massive stars are not formed in clusters, such as in the Galactic disk, but seem to be formed in (near) isolation with masses up to about $100 M_{\odot}$ or more. The arguments given above suggest that the formation of isolated massive stars in the Bulge of M51 could be due to the luminous central source, the low dust content, the small extinction and the consequent destruction of CO molecules in star forming clouds. The low cooling efficiency of such clouds then results in favouring the formation of massive stars.

This is analogous (but not the same) to the process of star formation in the very metal poor early Universe when the $\mathrm{C}$ and $\mathrm{O}$ abundance was very low and $\mathrm{H}_{2}$ was the dominant coolant. The similarity between star formation in the early Universe and the ongoing star formation near the nucleus of M51 is the absence of cooling by CO-molecules. The difference is the presence of cooling by atomic $\mathrm{C}$ and $\mathrm{O}$ near the nucleus of M51 but not in the metal-free early Universe. So the inefficiency of the cooling near the nucleus of M51 is less severe than in metal-free gas and the resulting stars may be less massive than those formed originally from metal-free clouds.

I suggest that we can learn about the formation of massive stars from metal-free clouds by studying the formation of massive stars in CO-free clouds. This process might take 
place when star formation occurs in regions of low extinction, $A_{V}<1$ or 2 mag, close to very luminous sources of radiation.

\section{Acknowledgements}

I thank my colleagues in this project: Nino Panagia, Martino Romaniello, Salvo Scuderi, Marco Spaans and Willem-Jan de Wit. I thank Volker Bromm and Jonathan Tan for useful discussions.

\section{References}

Aalto, S., Hüttemeister, S., Scoville, N.Z. \& Thaddeus, P. 1999, ApJ 522, 165

Abel T., Bryan, G. \& Norman, T.L. 2000, ApJ 540, 39

Abel T., Bryan, G. \& Norman, T.L. 2002, Science 295, 93

Barnes, J.E. 1998, in Interactions and Induced Star formation, eds. R.C. Kennicutt et al. (Berlin: Springer) 275

Bik, A., Lamers, H.J.G.L.M., Bastian, N., Panagia, N. \& Romaniello, M. 2003, AA 397, 473

Bromm, V., Coppi, P.S. \& Larson, R.B. 1999, ApJ 527, L5

Bromm, V., Coppi, P.S. \& Larson, R.B. 2002, ApJ 564, 23

Bromm, V. \& Larson, R.B. 2004, ARAA 42, 79

Clarke, C.J. \& Bromm, V. 2003, MNRAS 343, 1224

Haiman, Z., Thoull, A.A. \& Loeb, A. 1996, ApJ 464, 458

Haiman, Z. \& Loeb, A. 1997, ApJ 483, 21

Hernquist, L. 1990, in Dynamics and Interactions of Galaxies, ed. R. Wielen, (Heidelberg: Springer), 108

Kurucz, R.L. 1993, CD-ROM 13, ATLAS9 Stellar Atmospheres Programs (Cambridge: SAO)

Lamers, H.J.G.L.M., Panagia, N., Scuderi, S. et al. 2002, ApJ 566, 818

Leitherer, C., Schaerer, D., Goldader, J.D. et al. 1999, ApJS 123, 3

McKee, C.F. 1989, ApJ 345, 782

Mihos, J.C., Spaans, M. \& McCaugh, S.S. 1999, ApJ 515, 89

Norman, C. \& Spaans, M. 1997, ApJ 480, 145

Omukai, K. \& Palla, F. 2001, ApJ 561, L55

Omukai, K. \& Palla, F. 2003, ApJ 589, 677

Panagia, N., Lamers, H.J.G.L.M., de Wit, W.J. et al. 2001 in "The central kiloparsec of Starbursts and AGN", ASP Conf Series 249, 543

Salo, H. \& Laurikainen, E. 2000, MNRAS 319, 377

Scuderi, S., Panagia, N. Capetti, A., Romaniello, M. \& Lamers, H.J.G.L.M. 2005 (submitted)

Tan, J. \& McKee, C.F. 2004, ApJ 603, 383

Toomre, A. \& Toomre, J. 1972, ApJ 178, 623

Yoshida, N., Abel, T., Hernquist, R. \& Sugiyama, N. 2003, ApJ 592,645 